\title{
Successful treatment of calciphylaxis which was developed after kidney transplantation: a case report
}

\author{
Jun Young Lee ${ }^{1}$, Deok Gie Kim², Jae Won Yang ${ }^{1}$, Jae Seok Kim¹ , Byoung Geun Han ${ }^{1}$, Seung Ok Choi ${ }^{1}$ \\ ${ }^{1}$ Department of Internal Medicine-Nephrology, Yonsei University Wonju College of Medicine, Wonju, Korea \\ ${ }^{2}$ Department of Surgery-Transplantation, Wonju Severance Christian Hospital, Wonju, Korea
}

Background: Calciphylaxis is a rare dermatologic disease for kidney transplant (KT) recipients. We report successful treatment calciphylaxis skin lesions which was developed after KT.

Case report: Fifty-year-old male developed multiple ulcer-like skin lesions in both anterior tibia, end of the urethra, and anus. The patient had a history of liver cirrhosis, due to $\mathrm{C}$ viral hepatitis, benign prostate hyperplasia and received, 5 antigen mismatch, hypoxic brain death donor KT 15 months ago. In addition, he developed esophageal stricture 5 months after kidney transplantation. The patient's medications were tacrolimus, sirolimus for KT and furosemide for lower leg edema, and terazosin for benign prostate hyperplasia. The cause of esophageal stricture was chronic dysphagia the patient need balloon dilatation every 1 or 2 months. The patient's calcium level was $11.0 \mathrm{mg} / \mathrm{dL}$ and inactive parathyroid hormone level was $150 \mathrm{pg} / \mathrm{mL}$. In sonography there was one parathyroid $0.6 \times 0.5 \mathrm{~cm}$ well defined hypoechoic nodule in the left retro thyroidal area however there were no remarkably focal activities in the examined area of parathyroid and thyroid area in the parathyroid scan. We did a skin biopsy and reported that necrotic inflammatory exudate with dermal fibrosis, diffuse parakeratosis, orthokeratosis, irregular acanthosis, mild spongiosis, and upper dermal granulation tissue formation with dermal fibrosis. There was no other bacterial and viral infection evidence in culture and laboratory results. The wound was managed with the vacuum application, epidermal growth factor ointment, simazine, and push gel application every day. Cinacalcet hydrochloride and furosemide were applied to lower blood calcium levels and blood pressure. With those medications and wound management, ulcer-like skin lesions became smaller and eventually disappeared in both anterior tibia and end of urethra area after 6 months of treatment.

Conclusions: Calciphylaxis can develop in KT recipients and this might be associated with calcium level and could be successfully treated with hypercalcemia management. 